AperTO - Archivio Istituzionale Open Access dell'Università di Torino

Average daily ischemic versus bleeding risk in patients with ACS undergoing PCI: Insights from the BleeMACS and RENAMI registries

This is a pre print version of the following article:

Original Citation:

Availability:

This version is available http://hdl.handle.net/2318/1725725

since 2020-01-29T09:47:43Z

Published version:

DOI:10.1016/j.ahj.2019.10.001

Terms of use:

Open Access

Anyone can freely access the full text of works made available as "Open Access". Works made available under a Creative Commons license can be used according to the terms and conditions of said license. Use of all other works requires consent of the right holder (author or publisher) if not exempted from copyright protection by the applicable law. 


\section{Average daily ischemic vs. bleeding risk in patients with ACS undergoing PCl: Insights from the Bleemacs and Renami Registries}

Fabrizio D’Ascenzo, MD, Carloalberto Biolè MD, Sergio Raposeiras-Roubin, Federico Gaido, Emad Abu-Assi, Tim Kinnaird, Albert Ariza-Solé, Christoph Liebetrau, Sergio Manzano-Fernández, Giacomo Boccuzzi, Jose Paulo Simao Henriques, Christian Templin, Stephen B. Wilton, Pierluigi Omedè, Lazar Velicki, Ioanna Xanthopoulou, Luis Correia, Enrico Cerrato, Andrea Rognoni, Ugo Fabrizio, Iván Nuñez-Gil, Andrea Montabone, Salma Taha, Toshiharu Fujii , Alessandro Durante, Xiantao Song, Sebastiano Gili, Giulia Magnani, Michele Autelli, Federica Bongiovanni, Alberto Grosso, Tetsuma Kawaji, Pedro Flores Blanco, Alberto Garay, Giorgio Quadri, Dimitrios Alexopoulos, Berenice Caneiro Queija, PhD; Zenon Huczek, Rafael Cobas Paz, José Ramón González-Juanatey, María Cespón Fernández, Shao-Ping Nie, Isabel Muñoz Pousa, Masa-aki Kawashiri, Sara Rettegno, Diego Gallo, Umberto Morbiducci, Federico Conrotto, Alberto Dominguez-Rodriguez, Mariano Valdés, Angel Cequier, Andrés Iñiguez-Romo, Giuseppe BiondiZoccai MD, MStat, Gregg W. Stone and Gaetano Maria De Ferrari Prof..

Department of Cardiology, University of Torino, Italy.(FDA,CAB,PO,FC,MA,AG); Department of Cardiology, University Hospital Álvaro Cunqueiro, Vigo, Spain (SRR,AIR,EAA,BCQ,RCP). Cardiology Department, University Hospital of Wales, Cardiff, United Kingdom (TC,AG). Department of Cardiology, University Hospital de Bellvitge, Barcelona, Spain (AAS). Department of Cardiology, University Hospital Virgen Arrtixaca, Murcia, Spain (SMF), Division of Cardiology, Universitaspital, Zurich (CT,SG), Royal Brompton and Harefield Hospitals Trust and Imperial College, London, United Kingdom. Institute of cardiovascular Diseases, Vojvodina, Serbia (LV). University Patras Hospital, Athens, Greece. 'Interventional Unit, San Luigi Gonzaga University Hospital, Orbassano and Infermi Hospital, Rivoli (Torino), Italy.Catheterization Laboratory, Maggiore della Carità Hospital, Novara, Italy (AR). Department of Cardiology, S.G. Bosco Hospital, Torino, Italy. 'Department of Cardiology, Faculty of Medicine, Assiut University (ST). U.O. Cardiologia, Ospedale Valduce, Como, Ital (AD). Polito ${ }^{\mathrm{BIO}}$ Med Lab, Department of Mechanical and Aerospace Engineering, Politecnico di Torino (DG,UM). oDepartment of Cardiology, University Hospital from Canarias, Tenerife, Spain; University of Amsterdam, Academic Medical Center, Amsterdam. (JPH); Cardiovascular Institute of Alberta, Calgary (SBW); University Patras Hospital, Patras (DA), San Carlos Hospital, Madrid (ING); University Clinical Hospital, Santiago de Compostela (GRGJ); Anzhen Hospital, Beijing. (XS, SPN); Kerckhoff Heart and Thorax Center, Frankfurt (CL); University Graduate School of Medicine, Kyoto (TK); University Clinical Hospital, Warsaw (ZH); Tokai University School of Medicine, Tokyo (TF); Hospital Sao Rafael, Salvador(LC); University Graduate School of Medicine, Kanazaw (MAK); Department of Medico-Surgical Sciences and Biotechnologies, Sapienza University of Rome, Latina, and Department of AngioCardioNeurology, IRCCS Neuromed, Pozzilli, both in Italy (GBZ); Clinical Trials Center, Cardiovascular Research Foundation, New York, NY, United States; NewYork-Presbyterian Hospital/Columbia University Medical Center, New York, NY, United States (GWS) 


\section{ABSTRACT.}

Introduction. The risk of recurrent ischemia and bleeding after percutaneous coronary intervention (PCI) for acute coronary syndrome (ACS) may vary during the firstyear of follow-up according to clinical presentation, medical and interventional strategies.

Methods. BleeMACS and Renami are two multicenter registries enrolling patients with $\mathrm{ACS}$ treated with $\mathrm{PCl}$ and clopidogrel, prasugrel or ticagrelor. The average daily ischemic and bleeding risks (ADIR and $A D B R$ ) in the first year after $\mathrm{PCl}$ were the primary endpoints. The difference between ADBR and ADIR was calculated to estimate the potential excess of bleeding/ischemic events in a given period or specific subgroup.

Results. 19,826 patients were included. Overall, in the first year after $\mathrm{PCl}$ the ADBR was $0.008085 \%$, while ADIR was $0.008017 \%(p=0.886)$. In the first 2 weeks ADIR was higher than ADBR $(p=0.013)$, especially in patients with STEMI or incomplete revascularization. ADIR continued to be, albeit non-significantly, greater than ADBR up to the $3^{\text {rd }}$ month, while ADBR became higher, although not significantly, afterwards. Patients with incomplete revascularization had an excess in ischemic risk $(p=0.003)$, while non-STelevation-ACS patients (NSTE-ACS) and those on ticagrelor had an excess of bleeding ( $p=0.012$ and $p=0.022$ respectively).

Conclusions. In unselected ACS patients, ADIR and ADBR occurred at similar rates within 1 year after $\mathrm{PCl}$. ADIR was greater than ADBR in the first 2 weeks, especially in STEMI patients and those with incomplete revascularization. In the first year ADIR was higher than ADBR in patients with incomplete revascularization, while ADBR was higher in NSTE-ACS patients and in those discharged on ticagrelor. 


\section{INTRODUCTION}

Patients with acute coronary syndromes (ACS) treated with percutaneous coronary intervention $(\mathrm{PCl})$ have a substantial rate of recurrent ischemic and bleeding adverse events, in part due to the increased life expectancy which has raised the median age of patients presenting with ACS. Older patients present a larger rate of comorbidities such as malignancy, frailty and chronic kidney disease which increase both the ischemic and the haemorragic risk ${ }^{1,2}$. Conversely, technological improvements support the obtainment of satisfactory results with $\mathrm{PCl}$ even in challenging anatomies, although prolonged DAPT is often required ${ }^{3-7}$. Choosing among clopidogrel, prasugrel and ticagrelor treatments and related durations may depend on the relative ischemic and bleeding risks ${ }^{8-10}$.

Recently Giustino et al reported the average daily ischemic risk (ADIR) and average daily bleeding risk (ADBR) in different time intervals after $\mathrm{PCl}$ among patients with STsegment elevation myocardial infarction (STEMI) who were enrolled in the HORIZONSAMI trial, showing a greater risk of bleeding within the first 30 days and a greater risk of ischemia between 30 days and 1 year ${ }^{11}$. This study, however, was limited by strict inclusion criteria (only patients with STEMI), and exclusion of some high-risk patients, such as those with previous stroke or bleeding ${ }^{11,12}$. Consequently we reviewed the ADIR and ADBR in two large contemporary cohorts of ACS patients treated with clopidogrel, prasugrel and ticagrelor, with the aim of understanding the absolute and relative adverse events rates in a real life population ${ }^{13,14}$. 


\section{METHODS}

The study population was selected from the RENAMI and BleeMACS registries, with a total of 19,826 patients analyzed.

RENAMI (REgistry of New Antiplatelets in patients with Myocardial Infarction) included 4424 patients aged $\geq 18$ years with STEMI and NSTEMI, who underwent coronary angiography and $\mathrm{PCl}$ and were treated with DAPT using aspirin and either ticagrelor, prasugrel or clopidogrel. RENAMI patients were enrolled from 12 European centers between 2012 and $2016^{14}$.

BleeMACS (Bleeding complications in a Multicenter registry of patients discharged with diagnosis of Acute Coronary Syndrome) is an international multicenter investigator-initiated retrospective registry, made of 15,402 consecutive patients discharged alive after admission for ACS and treated with $\mathrm{PCI}$ during the index admission. BleeMACS registry is based upon patients from 15 tertiary hospitals in Europe, Asia, North and South America between 2003 and 2014 ${ }^{13}$. Both registries had no exclusion criteria. The institutional review board of each center approved participation in both BleeMACS and RENAMI registries.

Clinical data and follow-up. Clinical data (e.g. cardiovascular risk factors, clinical presentation), interventional data (e.g. access, extent of coronary disease and treatment), and outcomes were collected by a trained study coordinator in each center. Clinical assessments, ECG recordings and further instrumental examinations (when required) were performed periodically in every patient focusing on exact date of recurrent events.

Definition and end points. Bleedings events were defined as type 2-5 according to the Bleeding Academic Research Consortium (BARC) definition ${ }^{15}$. Ischemic events were 
defined as myocardial infarction (MI) and definite stent thrombosis (ST). Incomplete revascularization was defined as a final angiography result with coronary stenosis $\geq 70 \%$ in major epicardial vessels or stenosis $\geq 50 \%$ in the left main.

The ADIR and ADBR in the first year after PCI for ACS were the primary end point. The difference between average daily bleeding and ischemic risk was calculated, to detect the presence of a potential excess of bleeding/ischemic events in a given period or in a specific subgroup (sex, elderly people >=75 yo, type of P2Y12 inhibitor among clopidogrel, prasugrel, ticagrelor, type of infarction [STE vs. NSTE-ACS], completeness of revascularization, and history of malignancy).

Instantaneous daily bleeding and ischemic rates were calculated dividing the number of events occurring in a specific day post PCI for ACS by the number of exposed people on the same day. As the registries analyzed in this study take into consideration only single events, patients who had an event were excluded from the population at risk thereafter. The average risks were defined as the total number of events in that specific time interval divided by the total number of patient-days of follow-up, i.e., the total number minus loss at follow-up, deaths and people who already had an event. After calculation of daily risks, paired t test was applied to verify if there was a significant difference in term of ischemia/bleeding during the various time frames and subgroups. Statistical significance was defined as $p$ value $<0.05$

No extramural funding was used to support this work.

The authors are solely responsible for the design and conduct of this study, all study analyses and drafting and editing of the paper.

\section{RESULTS}


19,826 total patients with ACS were included. The average age of the patients was $63 \pm 12.5$ years, $23 \%$ were female and $25 \%$ had diabetes mellitus. Patients presenting with STEMI were 11,506 (58\%), 2366 (12\%) were treated with prasugrel, and 3356 (17\%) were treated with ticagrelor (Table 1).

Average ischemic and bleeding risk in the first year. There were 563 ischemic events and 567 bleeding events in our population in the first year of follow-up. The ADBR in the first year was $0.008085 \%$, the ADIR was $0.008017 \%$ (ADIR-ADBR=0.000068\%; $p=0.88$ ). The same results was observed in all the subgroups, except those with incomplete revascularization (Table 2 and Figure 1), in whom an excess in ischemic risk was noted (ADIR-ADBR=0.003264881\%; $p=0.003$ ), and NSTE-ACS patients and those taking ticagrelor, who showed an excess of daily bleeding risk (ADIR-ADBR=$0.001725756 \% ; p=0.012$ for NSTE-ACS, and ADIR-ADBR=-0.002356313\%; $p=0.022$ for ticagrelor use). There was no significant difference in the time distribution of bleeding risk in patients with NSTE-ACS and in ticagrelor patients, compared with the overall population.

Average ischemic and bleeding risk in the first month. In the acute phase ( $\leq 24$ hours), the ADIR was $0.0605357 \%$ and the ADBR was $0.025223 \%$, with an ADIR-ADBR $=$ 0.0353127. In the first week, there was a significantly higher ischemic risk compared to bleeding (ADIR-ADBR $=0.015170 \% ; p=0.048)$, due to an excess of ischemic events in the subgroups of patients with incomplete revascularization (ADIR-ADBR=0.043110\%; $p=0.018)$ and male patients (ADIR-ADBR=0.023384\%; $p=0.024)$.

In the first two weeks after ACS, in the entire population ischemic risk was higher than bleeding risk (ADIR-ADBR=0.011220\%; $p=0.013$ ), due to an excess of ischemic events in patients with incomplete revascularization (ADIR-ADBR=0.029703\%; $p=0.005$ ) 
and males (ADIR-ADBR=0.013108\%; $p=0.033$ ), but also in patients who were hospitalized for STEMI (ADIR-ADBR=0.018098\%; $p=0.02$ ) and patients taking Clopidogrel (ADIR$A D B R=0.013740 \% ; p=0.026)$.

This difference was no longer significant in the entire population when comparing risks in the first month (ADIR-ADBR=0.004909\%; $p=0.066$ ), but persisted in those with STEMI (ADIR-ADBR=0.010520\%; $\mathrm{p}=0.020$ ) and incomplete revascularization (ADIRADBR=0.018372\%; $p=0.004$ ) subgroups (Table 3 and Figure 2).

Average ischemic and bleeding risk from 2 to 12 months. After the first month, the rates of ADIR and ADBR in the overall population were essentially superimposable, without significant difference. ADIR tended to be greater than ADBR during the $2^{\text {nd }}$ and $3^{\text {rd }}$ months, while ADBR was more prevalent, although not significantly, from the $4^{\text {th }}$ to the $12^{\text {th }}$ months (Figure 3).

\section{DISCUSSION}

The analysis from this large-scale population of patients with ACS undergoing PCI highlighted: 1) an overall low risk of both ischemic and bleeding complications within the first year; 2) a significant excess of daily ischemic risk detected during the first two weeks, more pronounced in patients with STEMI, incomplete revascularization, and in patients taking clopidogrel as P2Y12 inhibitor; 3) declining trends toward greater ischemic risk for the first three months, whereas the bleeding risk demonstrated a non-significant trend to predominance after the $4^{\text {th }}$ month; 4) patients with incomplete revascularization presented significantly higher ADIR, while patients treated with ticagrelor and those with NSTE-ACS presented higher ADBR throughout the whole year. 
Optimizing the balance between ischemic and bleeding risk still represents challenge for physicians managing ACS patients with PCI. In a recent sub-analysis from the ACUITY trial ${ }^{19}$, recurrent MI was directly linked to mortality, especially for ischemic events occurring within 30 days after the index $\mathrm{PCl}$. Conversely, major bleeding in the same cohort was associated with increased mortality throughout the 1-year follow up.

In the present analysis of recurrent ischemic and bleeding risks after $\mathrm{PCl}$ in $\mathrm{ACS}$ in more than 19,000 patients, the ADIR was greater than ADBR in the first 2 weeks, especially in STEMI patients and in those with incomplete revascularization or treated with clopidogrel. In contrast, Giustino et $\mathbf{a}^{11}$ reported that both ADIR and ADBR were highest in the first 24 hours after PCI for STEMI in clopidogrel-treated patients, and then declined rapidly. Giustino et al ${ }^{11}$ also reported that the rates of ADIR and ADBR were similar in the first 24 hours; ADBR was greater than ADIR between 1 and 30 days; and then ADIR was greater than ADBR between 1 and 12 months. The discrepancy of our results from the literature ${ }^{11}$ may in part reflect differences in patients and types of ADP antagonists used. In-hospital mortality of STEMI patients is higher than NSTE-ACS (not included in the HORIZONS-AMI analysis ${ }^{11}$, but representing $42 \%$ of our patients), and comorbidities were more commonly present in our unselected patient population ${ }^{19}$. Moreover one third of our population received ticagrelor or prasugrel which, compared to treatment with clopidogrel, have been shown to reduce ischemic events particularly in the first $\mathbf{3 0}$ days, at the cost of a constant increase in bleeding through the first year ${ }^{20-23}$. Finally, the definition of AIDR and ADBR differed somewhat between HORIZONSAMI and the present study. Specifically, cardiac death was considered a component 
of ADIR in HORIZONS-AMI but not in our study, and ADBR was defined as TIMI major or minor bleeding in HORIZONS-AMI, vs. BARC types 2-5 in the present study.

In the present analysis, a declining trend of ischemic risk was noted after the first month, and an increased trend of bleeding after the $4^{\text {th }}$ month was observed. This held true for STEMI and NSTE-ACS patients. These data support the findings from the TOPIC trial $^{24}$, suggesting that de-escalating DAPT potency to clopidogrel after 3 months vs. continued use of prasugrel or ticagrelor may provide a net favorable balance of ischemia vs. bleeding.

Of note, the results of the analysis differ among specific patient cohorts including those with NSTE-ACS, incomplete revascularization, and with ticagrelor use. Complete revascularization in both STEMI and NSTE-ACS has been associated with improved event-free survival25-28, with reductions in both mortality and recurrent ischemic events. In large registries ${ }^{25,29}$, complete revascularization has been achieved in less than half of the patients, resulting in increased ischemia due to disease progression and thrombosis of non-culprit lesions ${ }^{30,31}$. The present findings suggest that potent P2Y12 inhibitors may be of particular benefit if complete revascularization is not achieved. Similarly, the increased bleeding risk in NSTE-ACS patients is related to comorbidities including renal insufficiency or malignancy ${ }^{19}$. The excess of bleeding risk on ticagrelor, most evident after the first 3 months ${ }^{20,22,23}$, and not described for prasugrel, is perhaps related to contraindication in patients $>75$ years old, $<60 \mathrm{~kg}$ in weight and with previous stroke for the latter, all of which have been associated with major bleeding.

Limitations could affect the generality of our findings. A major one is that patients were censored after occurrence of the first event, whereas the study by Giustino et al considered multiple events, which constituted $21.1 \%$ of all ischemic events and $15.9 \%$ of 
all bleeding events ${ }^{11}$. We did not consider the relationship between sequential ischemic and bleeding events. However, the only analysis of the first event provides clinically relevant insights since medical therapy is often individually tailored after the first bleeding or ischemic event. A limitation regarding P2Y12 analysis is surely the lack of randomization of drugs, however this is inherent in the observational layout of the study, and it can provide an insight on their real-world use. Moreover, increased risk of ticagrelor may be related to selection bias towards high risk patients, that is those with previous stroke or older than 75 years old The attributable risks of ischemic and bleeding events to subsequent mortality were not determined in our analysis, which would be helpful to weight the relative risks of each and the benefits of their prevention. Finally, no data about adherence to DAPT were available.

Conclusions. In a large series of unselected ACS patients treated with PCI, ADIR was more prevalent than ADBR in the first 2 weeks, especially in patients with STEMI and incomplete revascularization. In the first year ADIR was higher than ADBR only in patients with incomplete revascularization. Patients with NSTE-ACS and those discharged with ticagrelor had higher ADBR. These data emphasize the importance of considering the type of ACS presentation, the completeness of revascularization by $\mathrm{PCl}$ and the potency of the prescribed P2Y12 inhibitor used as part of a DAPT regimen to optimize the risks of bleeding and ischemia after $\mathrm{PCl}$. 


\section{References}

1. Moretti, C. et al. THE STORM (acute coronary Syndrome in paTients end Of life and Risk assesMent) study. Emerg. Med. J. EMJ 33, 10-16 (2016).

2. Yeh, R. W. et al. Population Trends in the Incidence and Outcomes of Acute Myocardial Infarction. N. Engl. J. Med. 362, 2155-2165 (2010).

3. D'Ascenzo, F. et al. Incidence and Management of Restenosis After Treatment of Unprotected Left Main Disease With Second-Generation Drug-Eluting Stents (from Failure in Left Main Study With 2nd Generation Stents-Cardiogroup III Study). Am. J. Cardiol. 119, 978-982 (2017).

4. Iannaccone, M. et al. Optical coherence tomography evaluation of intermediate-term healing of different stent types: systemic review and meta-analysis. Eur. Heart J. Cardiovasc. Imaging 18, 159-166 (2017).

5. Iannaccone, M. et al. Rotational atherectomy in very long lesions: Results for the ROTATE registry. Catheter. Cardiovasc. Interv. Off. J. Soc. Card. Angiogr. Interv. 88, E164-E172 (2016).

6. Forouzandeh, F. et al. Performance of J-CTO and PROGRESS CTO Scores in Predicting Angiographic Success and Long-term Outcomes of Percutaneous Coronary Interventions for Chronic Total Occlusions. Am. J. Cardiol. 121,14-20 (2018).

7. Armstrong, E. J. et al. Angiographic stent thrombosis at coronary bifurcations: shortand long-term prognosis. JACC Cardiovasc. Interv. 5,57-63 (2012).

8. Roe, M. T. et al. Prasugrel versus clopidogrel for acute coronary syndromes without revascularization. N. Engl. J. Med. 367,1297-1309 (2012). 
9. D'Ascenzo, F. et al. Impact of design of coronary stents and length of dual antiplatelet therapies on ischaemic and bleeding events: a network meta-analysis of 64 randomized controlled trials and 102735 patients. Eur. Heart J. 38, 3160-3172 (2017).

10. Mauri, L. et al. Twelve or 30 months of dual antiplatelet therapy after drug-eluting stents. N. Engl. J. Med. 371, 2155-2166 (2014).

11. Giustino, G. et al. Characterization of the Average Daily Ischemic and Bleeding Risk After Primary PCI for STEMI. J. Am. Coll. Cardiol. 70, 1846-1857 (2017).

12. Stone, G. W. et al. Bivalirudin during primary $\mathrm{PCl}$ in acute myocardial infarction. $N$. Engl. J. Med. 358, 2218-2230 (2008).

13. http://bleemacs.wixsite.com/registry.

14. https://renami.000webhostapp.com/.

15. Standardized bleeding definitions for cardiovascular clinical trials: a consensus report from the Bleeding Academic Research Consortium. - PubMed - NCBI. Available at: https://www.ncbi.nlm.nih.gov/pubmed/21670242.

16. Mehran R et al. Associations of major bleeding and myocardial infarction with the incidence and timing of mortality in patients presenting with non-ST-elevation acute coronary syndromes: a risk model from the ACUITY trial. Eur Heart J 2009;30(12):1457-66

17. Edmund Anstey D et al. Race and Sex Differences in Management and Outcomes of Patients After ST-Elevation and Non-ST-Elevation Myocardial Infarct: Results From the NCDR. Clin Cardiol. 2016 Oct;39(10):585-595.

18. Ellis CJ et al. All-Cause Mortality Following an Acute Coronary Syndrome: 12-Year Follow-Up of the Comprehensive 2002 New Zealand Acute Coronary Syndrome Audit. Heart Lung Circ. 2017 in press 
19. Moretti C et al. THE STORM (acute coronary Syndrome in paTients end Of life and Risk assesMent) study. Emerg Med J. 2016 Jan;33(1):10-6.

20. Wiviott SD et al. Prasugrel versus clopidogrel in patients with acute coronary syndromes. N Engl J Med. 2007 Nov 15;357(20):2001-15.

21. Wallentin $\mathrm{L}$ et al. Ticagrelor versus clopidogrel in patients with acute coronary syndromes. N Engl J Med. 2009 Sep 10;361(11):1045-57.

22. Antman EM et al. Early and late benefits of prasugrel in patients with acute coronary syndromes undergoing percutaneous coronary intervention: a TRITON-TIMI 38 analysis. J Am Coll Cardiol 2008;51:2028-2033.

23. Becker RC et al. Bleeding complications with the $\mathrm{P} 2 \mathrm{Y} 12$ receptor antagonists clopidogrel and ticagrelor in the PLATelet inhibition and patient Outcomes (PLATO) trial. Eur Heart J 2011;32:2933-2944

24. Cuisset $\mathrm{T}$ et al. Benefit of switching dual antiplatelet therapy after acute coronary syndrome: the TOPIC (timing of platelet inhibition after acute coronary syndrome) randomized study. Eur Heart J. 2017 Nov 1;38(41):3070-3078.

25. Quadri G et al. Complete or incomplete coronary revascularisation in patients with myocardial infarction and multivessel disease: a propensity score analysis from the "reallife" BleeMACS (Bleeding complications in a Multicenter registry of patients discharged with diagnosis of Acute Coronary Syndrome) registry. Eurolntervention. 2017 Jul 20;13(4):407-414.

26. Bravo CA et al. Complete versus culprit-only revascularisation in ST elevation myocardial infarction with multi-vessel disease. Cochrane Database Syst Rev. 2017 May 3;5:CD011986. 
27. Moretti $\mathrm{C}$ et al. Management of multivessel coronary disease in STEMI patients: a systematic review and meta-analysis. Int J Cardiol. 2015 Jan 20;179:552-7.

28. Sardella G et al. Single-Staged Compared With Multi-Staged PCI in Multivessel NSTEMI Patients: The SMILE Trial J Am Coll Cardiol. 2016 Jan 26;67(3):264-72.

29. Iqbal MB et al. The prognostic significance of incomplete revascularization and untreated coronary anatomy following percutaneous coronary intervention: An analysis of 6,755 patients with multivessel disease Catheter Cardiovasc Interv. 2017 Sep

30. Cheruvu PK et al. Frequency and distribution of thin-cap fibroatheroma and ruptured plaques in human coronary arteries: a pathologic study. J Am Coll Cardiol 2007;50: 940-9.

31. Iannaccone $\mathrm{M}$ et al. Prevalence and predictors of culprit plaque rupture at OCT in patients with coronary artery disease: a meta-analysis. Eur Heart J Cardiovasc Imaging. 2016 Oct;17(10):1128-37. 